\title{
Chemin de crête entre liberté de la personne et responsabilité de la société
}

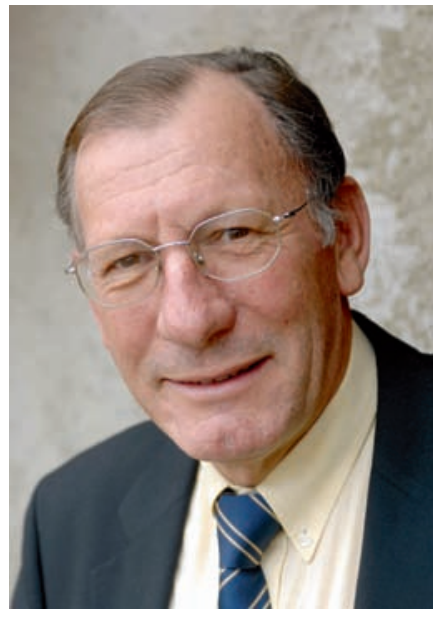

Jean Martin*

* Le Dr Jean Martin, membre de la rédaction, est ancien médecin cantonal vaudois et membre de la Commission nationale d'éthique.

1 Bosshard G. Sterbehelfer - eine neue Rolle für Europas Ärzteschaft? Bull Méd Suisses. 2008 89(10): 406-10.

2 Beutler D. Leben im Sterben. Bull Méd Suisses. 2008;89(10):411-5.

3 Kesseli B. Les médecins face à l'évènement ultime de la vie. Bull Méd Suisses. 2008;89(10):430.

4 Martin J. Assistance au suicide dans des situations médicalisées. Dans: Rehmann-Sutter C, et al. (éd.). Beihilfe zum Suizid in der Schweiz. Berne: Peter Lang; 2006. p. 295-307.

5 Académie Suisse des Sciences Médicales. Prise en charge des patients en fin de vie. 2004. www. samw.ch.

6 Commission nationale d'éthique. L'assistance au suicide. Prise de position no 9/2005. www.nek-cne.ch

7 Commission nationale d'éthique. Critères de diligence concernant l'assistance au suicide. Prise de position no 13/2006. www. nek-cne.ch.
Le numéro 10/2008 de ce journal inclut des contributions [1-3] en rapport avec l'assistance au suicide dans des situations «médicalisées»: quand il s'agit d'une personne gravement souffrante, sans perspectives de guérison et où le pronostic est sombre - que cette personne vive à domicile, dans un home pour personnes âgées ou à l'hôpital. La Suisse est dans une position particulière à cet égard, avec l'article 115 du Code pénal qui dispose que cette assistance n'est pas punissable sauf si elle est liée à un mobile égoïste [4]. L'Académie Suisse des Sciences Médicales a formulé à ce sujet des directives médico-éthiques [5] et la Commission nationale d'éthique (CNE) a présenté dans deux documents un cadre pour les professionnels et les établissements de santé $[6,7]$.

Dans nos sociétés, le fait qu'il n'y ait pas lieu de punir les suicidants est reconnu. On peut parler d'un droit à l'intention de se suicider (étant souligné qu'il s'agit d'un droit-liberté et que personne ne saurait exiger d'autres, par exemple de l'Etat, qu'ils mettent à disposition les moyens de le faire). Traduction de ce que, sous réserve d'actes illicites, l'Etat n'a pas à interférer dans la manière dont un individu gère son existence, même si ses choix apparaissent discutables ou sont réprouvés moralement par certains. Par ailleurs, il est clair que la médecine et la santé publique ont parmi leurs missions de faire œuvre de prévention du suicide. Mais, ici, comment nier des différences significatives entre le geste suicidaire de l'individu jeune ou adulte qui a devant lui un futur statistiquement long et qualitativement prometteur, et l'envie de voir sa vie se terminer d'une personne âgée, malade, qui a vécu l'essentiel de ce que peut offrir l'existence et pour qui le futur est fait de grande dépendance, de douleur, de pauvreté relationnelle?

Cela étant, il importe de discuter la dimension institutionnelle, publique, de la problématique. En home ou en hôpital, le suicide assisté diffère de la démarche limitée à deux acteurs et quelques proches qu'il est à domicile - même si on souhaite que les personnes aient les mêmes droits où qu'elles se trouvent. En institution sont forcément concernés des responsables (comité, direc- tion), les soignants, les corésidents de la personne qui entend mettre fin à ses jours. Ces aspects doivent être évalués en tenant compte à la fois de l'autonomie de l'individu et du respect dû aux autres. Dans les cantons de Vaud et de Genève notamment, les associations d'établissements médico-sociaux (homes) et les hôpitaux universitaires ont émis ces dernières années des documents équilibrés qui ont demandé un certain courage; documents qui, dans la clarté et dans un cadre défini, admettent que le suicide assisté y soit possible.

Question: une réglementation détaillée représente-t-elle une légitimation sociétale du suicide assisté? Tout en ayant personnellement une attitude d'ouverture, il me paraît que l'assistance au suicide doit garder, au plan de la collectivité, une dimension de transgression. Etant admis que cette transgression est compréhensible dans certaines situations considérées chacune dans ses caractéristiques spécifiques. Etant admis aussi, comme le demande la CNE [6], que «les professionnels de la santé ne doivent encourir aucune désapprobation morale ni aucune sanction professionnelle du fait de leur détermination, en conscience, en faveur ou contre l'assistance au suicide».

J'ai participé à un groupe de travail d'une fondation fournissant les soins à domicile dans notre canton, avec pour mission d'élaborer des recommandations pour le cas de demande d'accompagnement dans une situation d'assistance au suicide. Lors de l'adoption de ce document qui va dans le sens des textes émis par la CNE -, des médecins comme des élus ont insisté sur le fait qu'il convenait d'être attentif à ne pas donner l'impression à l'extérieur - et aux patients suivis - que le suicide devient une option comme les autres, une chose «normale». Tout en respectant la liberté des personnes, il importe qu'institutions et pouvoirs publics évitent toute démarche susceptible d'être interprétée comme une caution du suicide, voire une propagande! Il $\mathrm{y}$ a là, conceptuellement et très pratiquement, un chemin de crête éthique qui doit recevoir une attention adéquate.

Jean Martin 